\title{
From Adaptive Reasoning to Cognitive Factory: Bringing Cognitive Intelligence to Manufacturing Technology
}

\author{
Indar Sugiarto $^{1, a}$, Cristian Axenie ${ }^{2, b}$, Jörg Conradt ${ }^{2, c}$ \\ ${ }^{1}$ Department of Electrical Engineering, Petra Christian University, Indonesia \\ ${ }^{2}$ Neuroscientific System Theory, Technische Universität München, Germany \\ indiapetra.ac.id, ${ }^{\mathrm{b}} \mathrm{cristian} \cdot \mathrm{axeniedtum.de,}{ }^{\mathrm{c}} \mathrm{con}$ radtetum.de
}

\begin{abstract}
There are two important aspects that will play important roles in future manufacturing systems: changeability and human-machine collaboration. The first aspect, changeability, concerns with the ability of production tools to reconfigure themselves to the new manufacturing settings, possibly with unknown prior information, while maintaining their reliability at lowest cost. The second aspect, human-machine collaboration, emphasizes the ability of production tools to put themselves on the position as humans' co-workers. The interplay between these two aspects will not only determine the economical accomplishment of a manufacturing process, but it will also shape the future of the technology itself. To address this future challenge of manufacturing systems, the concept of Cognitive Factory was proposed. Along this line, machines and processes are equipped with cognitive capabilities in order to allow them to assess and increase their scope of operation autonomously. However, the technical implementation of such a concept is still widely open for research, since there are several stumbling blocks that limit practicality of the proposed methods. In this paper, we introduce our method to achieve the goal of the Cognitive Factory. Our method is inspired by the working mechanisms of a human's brain; it works by harnessing the reasoning capabilities of cognitive architecture. By utilizing such an adaptive reasoning mechanism, we envision the future manufacturing systems with cognitive intelligence. We provide illustrative examples from our current research work to demonstrate that our proposed method is notable to address the primary issues of the Cognitive Factory: changeability and human-machine collaboration.
\end{abstract}

Keywords: Adaptive Reasoning, Cognitive Factory, Brain-inspired Processing.

\section{Introduction}

The challenges of the $22^{\text {nd }}$ century manufacturing system rely on the dynamic interaction between welldefined manufacturing processes and the adaptability of tools developed by engineers. The tools are not only capable of handling high speed and high precision production processes, but they are also expected to be able to cope with uncertainty and risk introduced in a dynamic environment [1-3].

In a dynamic environment, the production tools work under direct supervision of humans or they share the working place with humans. In both cases, the interaction of both production agents (machinery and humans) may produce interdependency that leads to a cooperation scenario. Within this scenario, the paradigm of reconfigurable manufacturing was developed [4-5].

In a reconfigurable manufacturing, the system behavior is changed as a result of an applied new system configuration [6-8]. Hence, this reconfigurable paradigm is not only intended to supplement the existing flexible manufacturing system with agility and adaptability, but also with a changeability attribute. This changeability attribute provides a systematic treatment to deal with uncertainty in a manufacturing environment [9].

Many researchers see this changeability attribute as a characteristic to accomplish early and foresighted adjustments of factory's structures and processes economically on all levels. In general, the changeability always requires system configurations that consist of exchangeable compo- nents. In this circumstance, the changeability of manufacturing behavior can be achieved by adaptively reconfiguring the production tools (of both hardware and software), which leads to the development of a reconfigurable system.

The concept of reconfigurable machine tools requires that the interfaces between the elements of the manufacturing system should be kept at minimum in order to enable reintegration. Practically, this can be achieved through selfsustaining mechatronic configurable modules that contain all components needed for a satisfactory function. One potential solution of this practicality can be attained by reducing the number of interfaces to a bus system for communication and a bus system for the energy supply. However, such an approach might not be a general solution and suffer its immediate drawback especially with regard to the limitation of the existing machine tools or plants.

In this paper, we propose a novel solution for manufacturing agents by applying a more flexible treatment through learning mechanisms. Our approach comes from the insight that the highest degree of changeability is still reached by human workshops with skilled workers and their cognitive capabilities, which enable them to react to changes, perceive their environment, plan their next actions, and know what they are doing.

To achieve such a level for a production system, we need to look for a new strategy that puts production planning and automation in a cognitive manner. This idea, which leads to the proposal of a Cognitive Factory, is further examined by several research institutes and universities. Basically, the Cognitive Factory is a form of Cognitive 
Technical Systems (CTS), which enables factory environments to react flexibly and autonomously to changes, similar to human operated facilities [10-12]. The main goal of such an intelligent facility is to increase the autonomy of manufacturing elements and to enhance their changeability. It utilizes some techniques of Artificial Intelligence (AI) and Cognitive Engineering (CE) by introducing a cognitive architecture which comprises knowledge models, methods for perception and control, methods for planning, and a cognitive perception-action loop [13-14].

Such reasoning tasks can be modeled systematically using biologically inspired cognitive architectures, and we propose to use brain-inspired information processing to conceive such models. By utilizing learning paradigms on the model, the required controller elements can be developed easily using the current know-how of the 'state-of-theart' of control systems. In addition, our approach will reinforce the self-adapting control systems with mechanisms for monitoring and identification of mechanical modules, and an online re-configuration for the automatic adaptation.

This paper is presented as follows. In section two, we describe the fundamental concept of brain-style information processing for cognitive embodiment that plays an important role in a reconfigurable manufacturing system. In section three, we demonstrate how we implement two sample cognitive architectures as the basis for developing and empowering a Cognitive Factory. In section four, we discuss some important aspects regarding the achievements of our methods. Finally, we conclude our work in section five and explain further direction of our research.

\section{Brain-inspired Processing with Cognitive Architecture}

The main theme of this paper is how we employ adaptive reasoning for a Cognitive Factory. By definition, reasoning is a mechanism of making sense of data by applying computational logic and algorithms based on new or existing information. We employed fundamental concepts developed in neuroscience to gain some insight on the brain-inspired processing mechanism. As a result, we developed networks that can be used to construct a cognitive architecture that can be used to empower a Cognitive Factory. This section describes the fundamental concepts of our method.

\subsection{From CTS to Cognitive Factory}

We argue that the primary requirement to address changeability of reconfigurable manufacturing systems is the transfer of cognitive skills, which are attributed to intelligent animals and humans, to technical systems such as robots, manufacturing systems and vehicles. This will take a form of reasoning: such technical systems can perceive and react appropriately to their environment, and even can cooperate with people in a way that humans can cope with, both physically and intellectually. These capabilities can be acquired through learning processes. Hence, cognitive technical systems (CTS) can be built as information processing systems that perform cognitive control and have cognitive capabilities. Figure 1 shows how the reasoning as a close perception-action loop is conceived in a cognitive architecture.

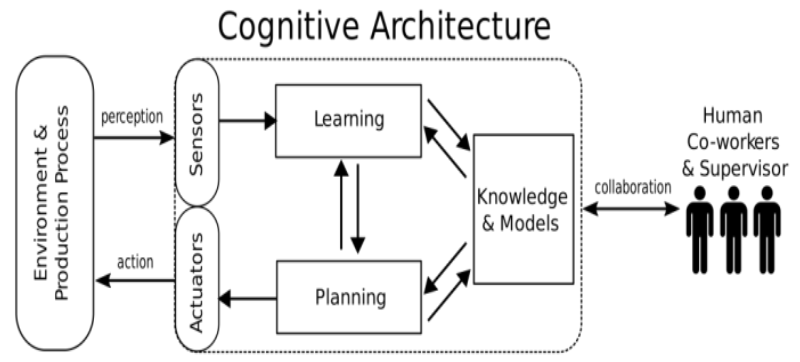

Figure 1. Reasoning through a cognitive architecture

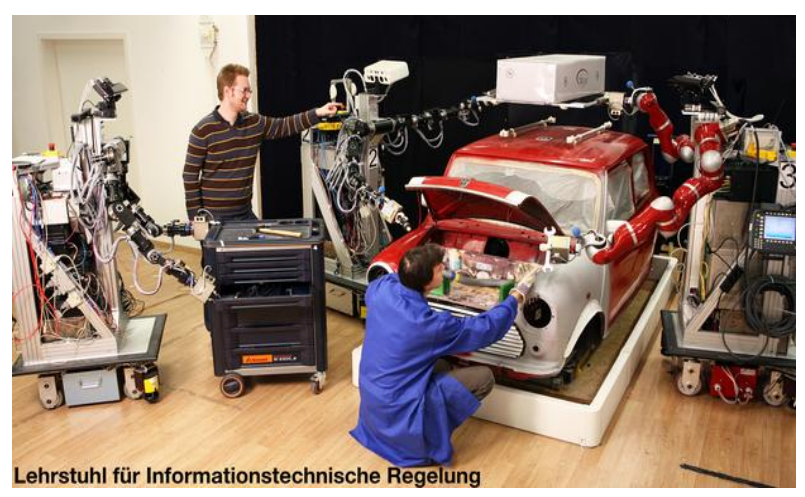

Figure 2. The seamless interaction between humans and machines will be a key to success of a Cognitive Factory (source: http://www.ei.tum.de/en/research/coc-roboticsautonomy-and-interaction/)

To deliver this concept in reality, the German Cluster of Excellence called CoTeSys ("Cognition for Technical Systems") was founded. This research is coordinated by Institute of Automatic Control Engineering (Lehrstuhl für Steuerungs- und Regelungstechnik) at Technische Universität München (TUM) as a close collaboration between scientists from various disciplines connecting neurocognitive and neuro-biological foundations to engineering sciences. CoTeSys investigated cognition for technical systems such as vehicles, robots, and factories. One milestone of this project is the concept of Cognitive Factory that uses cognitive architectures as the foundation of a manufacturing system. Within this framework, constructive collaboration between humans and machines becomes one important aspect (see Figure 2).

The Cognitive Factory combines the advantages of automated systems (e.g. low costs, high quality, high efficiency and low manufacturing times) with the flexibility, adaptability and reactivity of common human workshops. The position of this Cognitive Factory within the domain of manufacturing systems is depicted in Figure 3.

With cognitive architecture, a factory system can learn new situation-specific models of production steps. It can also estimate the state of manufacturing processes and then dynamically reschedule the production steps in order to compromise disturbances as well as to give a response to dynamically-changed objective functions of the process. Such a cognitive capability is argued to be the best mechanism that provides human process planners with much more informative models of production processes that are learned from experience. This in turn will lead to the best practice of human-machine collaborations. 


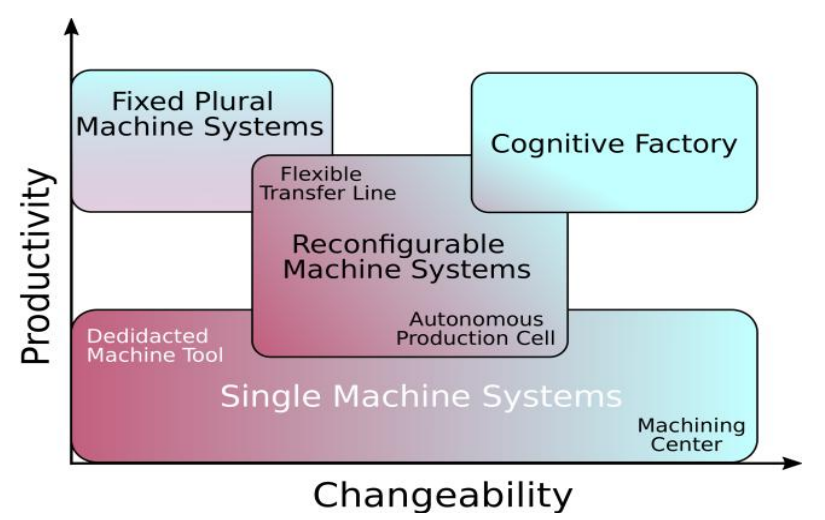

Figure 3. Cognitive Factory as a new framework in the domain of manufacturing system

In order to achieve such excellent goals, we need to embody the cognitive intelligence itself to the manufacturing tools. There are several strategies that can be applied to obtain a cognitive architecture for the embodiment process. In our work, we are interested to investigate the brain-style information processing to apply the cognitive intelligence to the elements of a Cognitive Factory. The idea of brain-style information processing can be applied in many aspects, but in this paper we only cover the most two fundamental concepts: cortically inspired network and brain-connectome inspired network. Figure 4 depicts our interpretation of brain mechanism based on neurological data collected across cortex area.

\subsection{Cortically-inspired Network for Information Processing}

In neuroscience, cortex is a part of the mammalian brain (including human brain), that involves in higher functions such as sensory perception, generation of motor commands, spatial reasoning, conscious thought and language. Reasoning mechanisms in the cortex are very different with the standard computing mechanisms in modern digital computer systems. Computers use re-programmable, highperformance CPUs to process data fetched from and stored to memory, whereas in brains, neural processing and synaptic data storage are completely inter-meshed, with each cortical area being responsible for both memory and processing. The cortical circuitry can be mimicked in a technical system, for example, to perform egomotion estimation.

It is commonly agreed that the brain contains areas specialized for processing different types of information incoming from sensors [15]. A major determinant for a brain area's ability to process a certain type of information is the input it receives. Cortical areas, through their coordination dynamics, are thought to rapidly resolve a large number of mutually imposed constraints, leading to consistent local states and a globally coherent state of cognition [16].

The unique processing characteristic of each cortical area is defined in terms of the area's interactions with the other areas [17]. Hypotheses from cortical inter-areal coordination studies support evidence that these areas aim to reach a consensus and maintain mutually consistent information with the others resolving coherence or incoherence relations (i.e. constraints). There is strong behavioral and physiological evidence that the brain both represents probability distributions and performs probabilistic inference [18]. Furthermore, neurobiological evidence supports the view that elementary sensory representation and functions are localized in discrete areas, whereas complex functions are processed in parallel in widespread networks [19].

Large-scale cortical networks provide a framework to integrate evidence from neuroanatomical and neurophysiological studies on distributed information processing in the cerebral cortex. Elementary sensory processing functions are localized in discrete recurrently interacting cortical areas, whereas complex functions (e.g. cue integration) are processed in parallel involving large parts of the brain.

Based on those findings, we develop our corticallyinspired network that can be used to empower an intelligent system. In section 3.1 we describe one particular example of application derived from our network.

\subsection{Brain-connectome-inspired Network for Cognitive Architecture}

For more than 50 years since its initial booming, Artificial Intelligence (AI) has attracted a lot of interdisciplinary
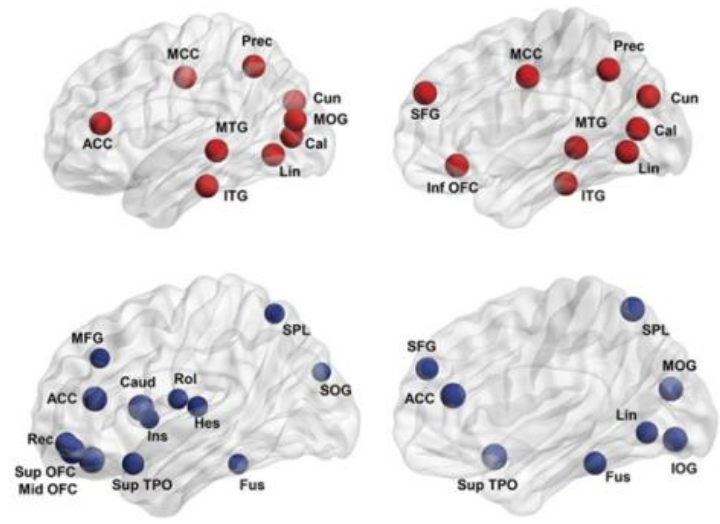
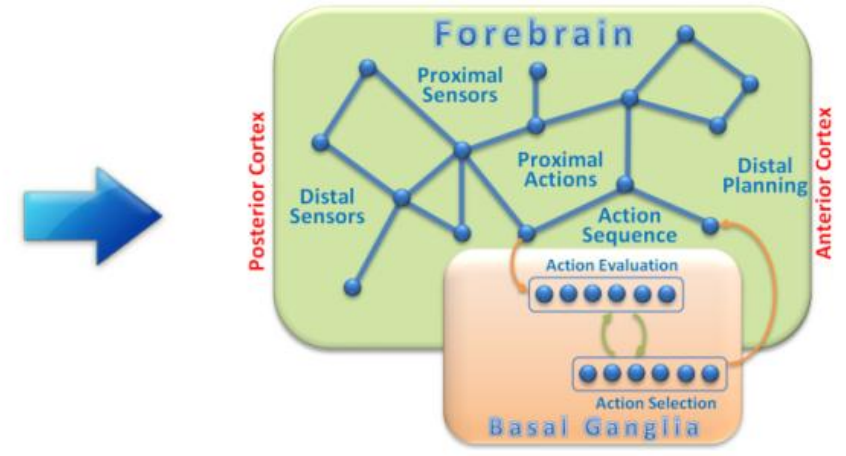

Figure 4. General overview of brain graph showing high level connection between parts in human's brain (the left figure is adapted from [23]) 
researchers to uncover one of the most fundamental challenges in science and technology: how does intelligence form? Within this domain, many AI researchers explore the cognitive capabilities such as perception, reasoning, planning, and learning with a long term goal of turning technical systems into systems that "know what they are doing" [13]. Technical systems with cognitive capabilities will be much easier to interact and cooperate with humans, and are expected to be more robust, flexible, and efficient when working in a dynamic environment such as human living space. Not surprisingly, this in turn leads to the development of the abstract concept of embodied mind.

One source of inspiration for the embodiment theory has been the research in cognitive neuroscience. The idea of the embodied mind starts to grow and becomes one of the intense debates within the field. Neuroscientists discuss how both our neural and developmental embodiment shapes our mental and linguistic skills. The degree of thought abstraction has been found to be associated with physical distance which then affects associated ideas and perception of risk [20]. They also explain the idea of embodied

\section{Neurological Data/Experiment}
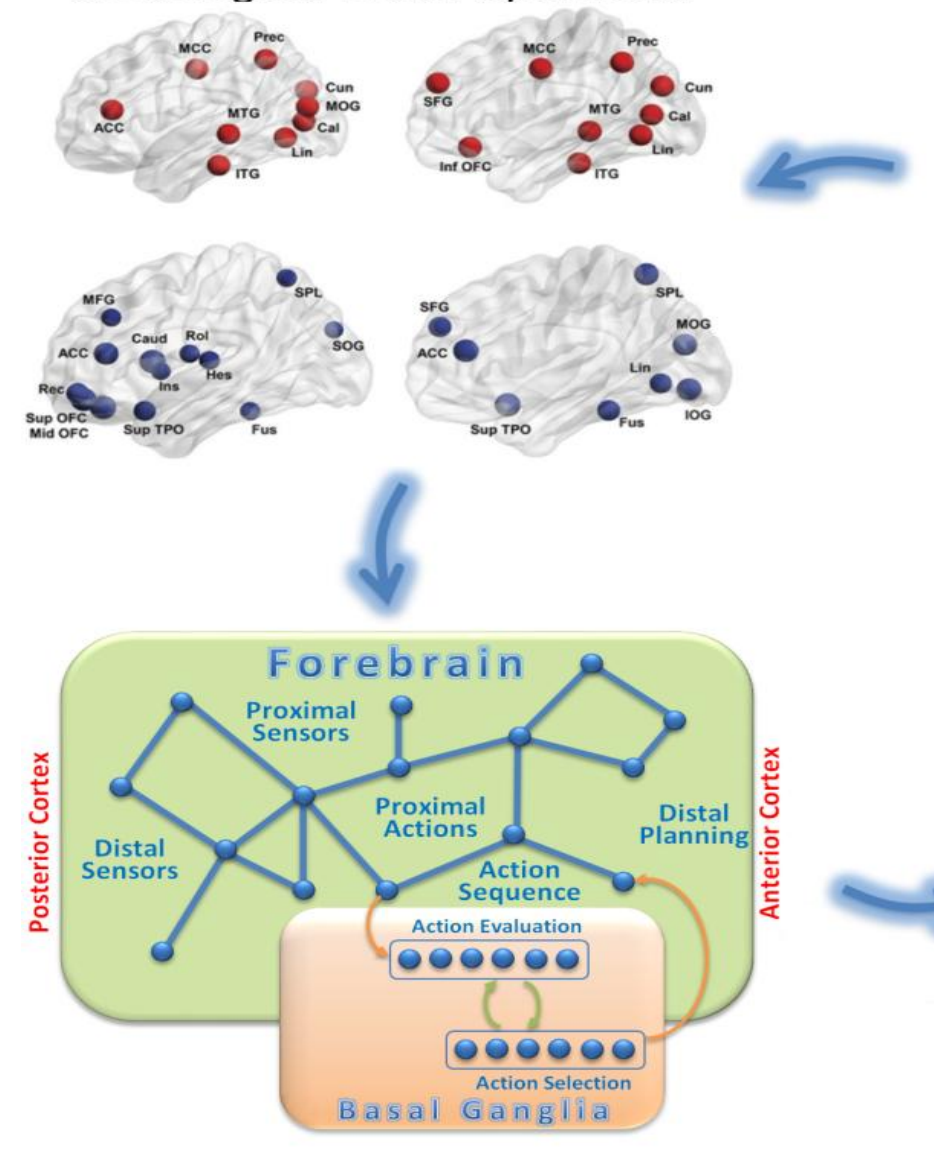

Brain Graph cognition in terms of dynamical systems theory which leads robotic experts to explore the domain of imitation learning [21-22].

In our work, we are interested to explore the idea of transforming brain neurological data into a network of interconnected nodes. This is the fundamental concept of Brain Graph theory [23]. A Brain Graph is a model of the connectome of a nervous system, which interconnects processing nodes through a set of communication edges. A connectome is a complete point-to-point spatial connectivity of neural pathways in the brain which represents a comprehensive map of neural connections in the brain [24]. Computational neuroscientists build these brain graph models using topological and geometrical approaches that demonstrate both structural and functional organization of the human brain [25]. In such Brain Graphs, nodes represent collections of similar neurons (in terms of structure and function), while edges represent structural - and hence functional - connections between those nodes.

Such a representation was originated from the research domain of Graph Theory, and shares many similarities with

\section{Hardware \& Software for Manufacturing Processes}
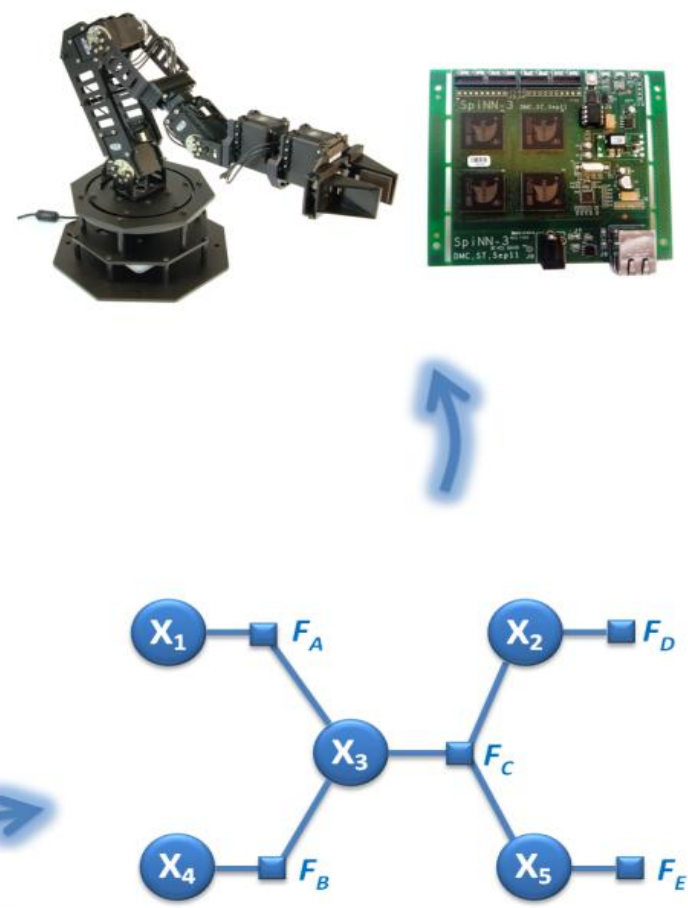

Cognitive Architecture

Figure 5. How we relate neuroscience, artificial/cognitive intelligence and robotics. We learn from neuroscience, how the cognitive intelligence is emerged; we learn from artificial intelligence (machine learning), how we can model it; we then apply our model in robotics applications that are suitable in a Cognitive Factory environment. We hope that our work may also provide valuable feedback and insight for our colleagues in neuroscience on how the cognitive intelligence should be studied further. 
probabilistic graphical models: (a) nodes are computational units within a distributed system that are functionally separable from each other; and (b) interactions between such nodes become continuously less meaningful the more similar the nodes are to each other.

In machine learning, probabilistic graphical models are used for computing uncertainty and for the generation of actions based on perception, which provides a unified framework for graph theory and probabilistic reasoning in complex real-world settings. This has led our work to develop a framework to generate intelligent behavior in a fashion similar to neural computation in the brain: a massively parallel distributed computing system, in which overall performance comes from independent computation of local units that communicate with a subset of neighboring nodes.

It is believed that cognitive intelligence can naturally be explained if human cognitive representations are understood to be structured like graphical models [26]. In our research, we model Brain Graphs for sensory perception and actuator action generation in the setting of autonomous robots that interacts in its environment (see Figure 1). We investigate Factor Graphs (a subclass of Graphical Models) to represent relations between observable sensory signals, possible hidden states, and desired robot actions. Transforming brain graphs into factor graphs can help the Machine Learning community to understand the technical constraints which make those brain graph models more applicable in technical systems. Figure 5 gives a notion of contribution of our work in related fields.

In the field of human physiology, it is a straightforward observation that humans can easily change from task to task without too much effort, revealing the fact that humans have a long history of adapting the model-based learning features. These model-based features are also believed by scientists, leading to the conclusion that intelligent mammals rely on their own internal models in order to generate their actions. While conventional robotics rely on manually generated models that are based on human insights into physics, it is also predicted that future autonomous, cognitive robots need to be able to automatically generate models that are based on information perceived by the robots [22].

In our work, we extended our basic factor graph framework into a dynamic one that is suitable for modelbased learning. We argue that model-based learning using factor graphs is one of important ingredients to create adaptive agents as the basis for reconfigurable manufacturing tools. This is because a factor graph can be regarded as a unified approach of both directed and undirected graph theory. This unified approach is an ideal solution for dealing with uncertainty: something that changeability is striving for. In section 3.2 we provide an example of how to use a factor graph for model-based learning to teach a robot to imitate human motions.

\section{Illustrative Examples}

The brain-inspired computing is a new fascinating approach that originates from the field of computational neuroscience. In this section, we give two examples of how we develop a cognitive architecture that functions as a base for a more complex system such as a Cognitive Factory.

\subsection{Cortically-inspired Sensor Fusion Network}

As an intelligent system, a manufacturing tool is expected to react intelligently in a dynamic environment. This action includes the self-localization of the agent moving in a factory working space. How can we apply cognitive intelligence on such an agent? One possible scenario is by utilizing egomotion estimation based on the theory of cortical networks [27].

Egomotion or self-motion refers to the combined rotational and translational displacement of a perceiver with respect to the environment. During motion, organisms build their spatial knowledge and behaviors by continuously refining their internal belief about the environment and own state [28]. During its development, the biological nervous system must constantly combine various sources of information and moreover track and anticipate changes in one or more of the cues. Furthermore, the adaptive development of the functional organization of the cortical areas seems to depend strongly on the available sensory inputs, which gradually sharpen their response, given the constraints imposed by the cross-sensory relations [29].

One important aspect that needs to be addressed on egomotion estimation is how precise egomotion perception can be obtained given the complex multisensory environment. We solve this by trying to disambiguate the complex and global representation of the environment. In this scenario, all cues are combined in an informative and plausible way that leads to a sensor fusion strategy. Sensor fusion is a process that influences major aspects of perception, cognition and behavior in both physical and artificial systems [30]. The primary objective is to align the reference systems of the different congruent and redundant sensory cues.

Our work is inspired by the distributed processing paradigm of the humans' brain. Studies in Neuroscience reveal that the brain is capable of multimodal learning and robustly adapting to under-constrained or conflicting sensory inputs. The brain can also maintain and continuously refine its internal belief about the environment. We implemented this insight into a cortically-inspired network for egomotion estimation. The main architecture of our network for egomotion estimation is depicted in Figure 6. There is no explicit input or output in/from the network and sensor data just mildly influence the activity in the network. Based on the embedded relations, the network is able, in the absence of one or more sensors, to infer the missing quantities.

The architecture shown in Figure 6 uses a distributed network in which independent neural computing nodes obtain and represent sensory information, while processing and exchanging exclusively local data, to infer an estimate of robot orientation and position. This model is inspired by the neural processing paradigm, where cortical areas involved in sensory processing assume rapid resolution of a large number of mutually imposed constraints (i.e. coherence/incoherence relations), leading to a globally coherent estimate of the percept. 


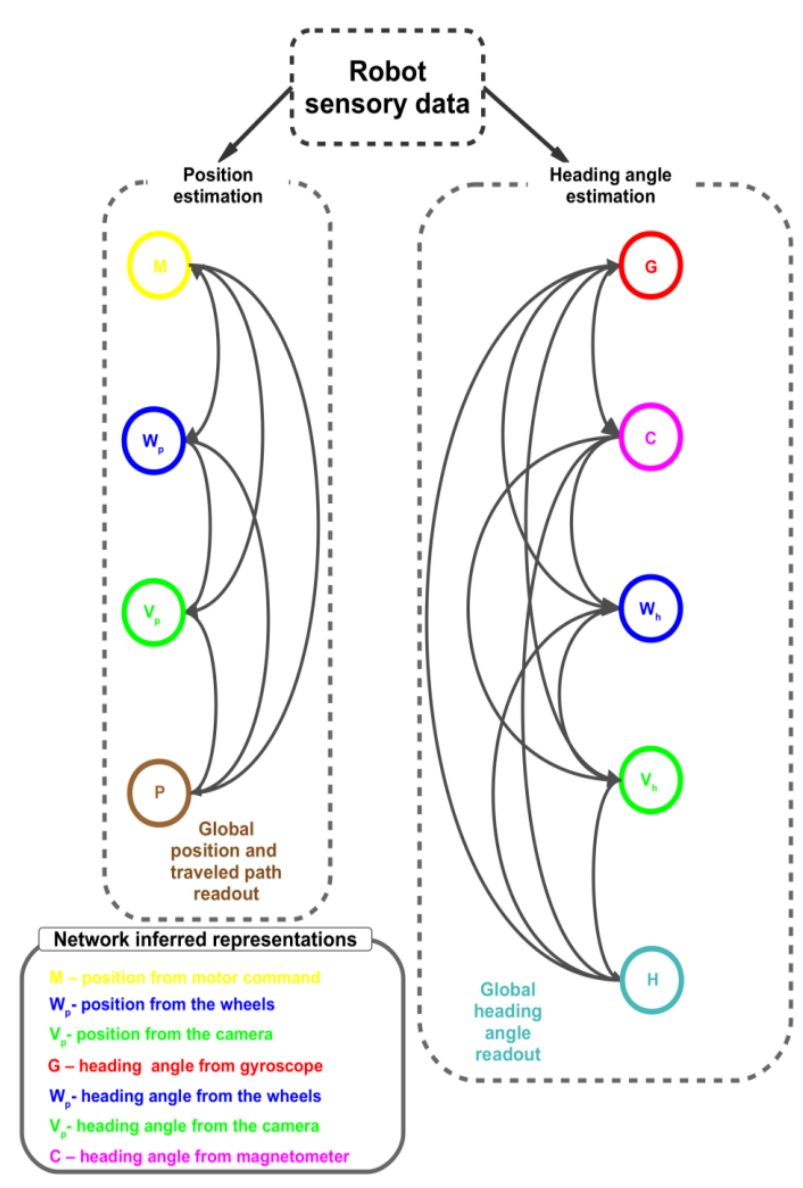

Figure 6. Network architecture for egomotion estimation. The distributed network is composed of interconnected subnetworks for heading and position estimation [27].

\subsection{Model-based Mobile Manipulator}

Programming the robot to carry certain task in a factory or in a daily life service is tedious work. The conventional method will usually involve precise mathematical formulation of the task followed by some fine-tuning parameters of the preprogrammed robot system. Even in a simple robot system, this routine will be cumbersome in a frequently changing tasks setting. This motivates many researchers in $\mathrm{AI}$ and robotic systems to find a more compliant/relaxing way to handle this situation. The idea is simple: teach the robot to generate motions based on human experience.

As an example test case, we developed a robotic application in a placing-an-object scenario. In this scenario, the robot moves an object from one point to another point in space where the distance may be far, so that the robot's arm is unable to put the object directly at the destination point. For this scenario, we developed a mobile manipulator - a hybrid robotic system, where a robotic arm is installed on top of a mobile robot, as shown in Figure 7.

This hybrid system is challenging because the robot should maintain its stability while moving (e.g. by aligning the arm so that the overall center-of-mass will be close to the center of the whole body of the robot). For our initial experiment purpose, the robot operation did not start by picking up an object. Instead, the object is placed into the gripper of the robotic arm.

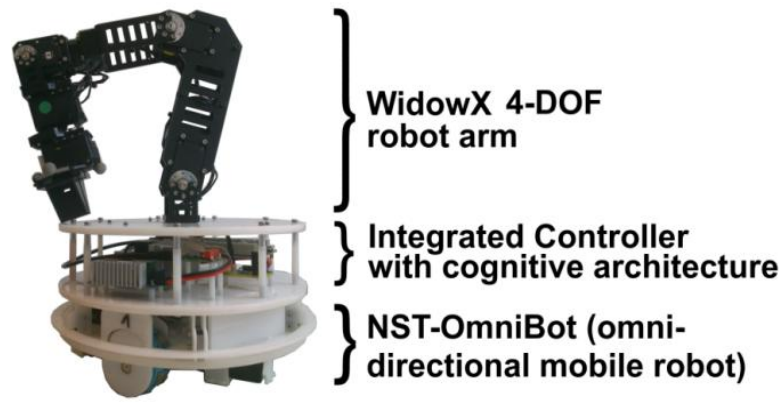

Figure 7. The mobile manipulator developed for the test case of our model-based learning method. It is composed of two subsystems: a 4-DOFs robotic arm and a mobile platform.

We defined a session as a single placing-an-object task. In the beginning of the session, the original coordinate of the object's center and the goal position are given. If required, the robot should move to the point where the arm is able to reach the object. When the robot is in the right position, the robot lifts the object and moves the arm, so that its overall center-of-mass is in a stable position. The robot then approaches the destination position. When it is close enough, the arm is moved (i.e. stretched), so that the object can be placed at the goal position. In the future, we plan to extend this motion with the localization task described in section 3.1. To apply model-based learning, we proposed a model shown in Figute 8.

The upper level of the model shown in Figure 8 is a model of a task constrained control of our mobile manipulator. In this model, we define several discrete variables (shown in boxes) as well as real-valued variables (shown in circles). In our notation, a task $\mathbf{T}=$ \{extend, rextend\} refers to a "basic task" that the robotic arm should do; that is, either extending or retracting its arm. The position of the end-effector will be determined by the variable $\mathbf{G}$, which indicates the target/goal position, and the current position of the mobile robot M. During the movement, the arm's orientation will be determined by the task constraint $C_{l}=$ fflat, - flat $\}$. The flat pose means that the arm should keep its joints configuration to be $0^{\circ}$ in total, so that the end-effector will always be in a horizontal position. The non-flat pose means that the arm can use all possible joints configurations to reach the target, allowing it to have the longest reaching posture. The reachability indicator $\mathbf{R}=$ \{reachable, reachable $\}$ determines whether the mobile robot should move (further toward or away to/from the object), or stay at the current position. It also determines whether the mobile robot should perform the rotation in order to align itself in-line with the object (positioned straight directly toward the object), or stay still. Hence, the value of this variable will depend on the position of the object along with its goal position, the current pose of the mobile robot, and the current pose of the robotic arm. In addition, we need to inform the variable $\mathbf{R}$ about the physical constraint (e.g. the diameter) of the mobile robot via the variable $C_{2}$.

The robot then creates the skill model using some learning algorithm that exploits the statistical regularities across multiple observations [31]. Figure 9 shows the basic principle of learning new skill using the programming by demonstration $(\mathrm{PbD})$ paradigm. 


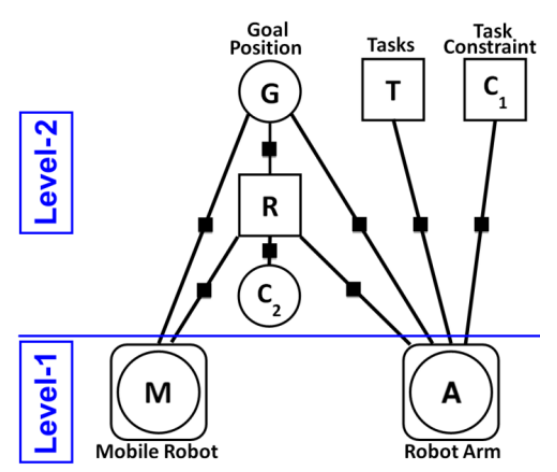

Figure 8. The graphical model of our mobile manipulator shown in Figure 7

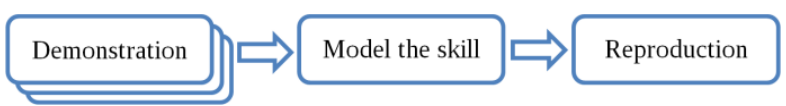

Figure 9. The conceptual principle of model-based learning using programming by demonstration

\section{Experimental Results and Discussion}

In section 3.1 we described one example application of the cortical network for egomotion estimation. In order to test our proposed model, we applied it for a quadrotor 3D egomotion estimation and extended it with learning capabilities, briefly depicted in Figure 10. This scenario is based on our previous work in [32]. In this scenario, a quadrotor hovers (remote controlled) in an uncluttered environment, while an overhead camera system keeps track of its position and orientation.

After the flight, preprocessed data from the available sensors (i.e. gyroscope, accelerometer and a magnetic sensor) are fed to the model to extract the relations between the sensors for each of the three degrees of freedom (i.e. roll, pitch and yaw). Figure 11 presents a decoupled view for each degree of freedom, depicting the learned relations and estimation accuracy.

We observe in Figure 11 that the learned relations resemble the nonlinear functions (i.e. arctangent) used in typical modeling approaches, although preserving irregularities in the cross-sensory relations. For roll estimation, the network learns the relation between net rotational acceleration provided by the accelerometer and the absolute roll angle estimate provided by the gyroscope. For pitch estimation, the network extracts the nonlinear dependency between the accelerometer data and the gyroscope data. For yaw estimation, the network uses the gyroscope absolute angle and the magnetometer contribution, based on magnetic field readings on the other two axes.
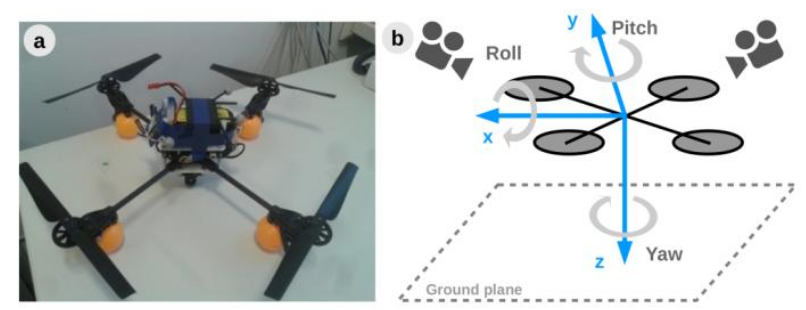

Figure 10. Experimental setup used in [32]: a) Quadrotor platform; b) Reference system alignment and ground truth camera tracking system
Network architecture

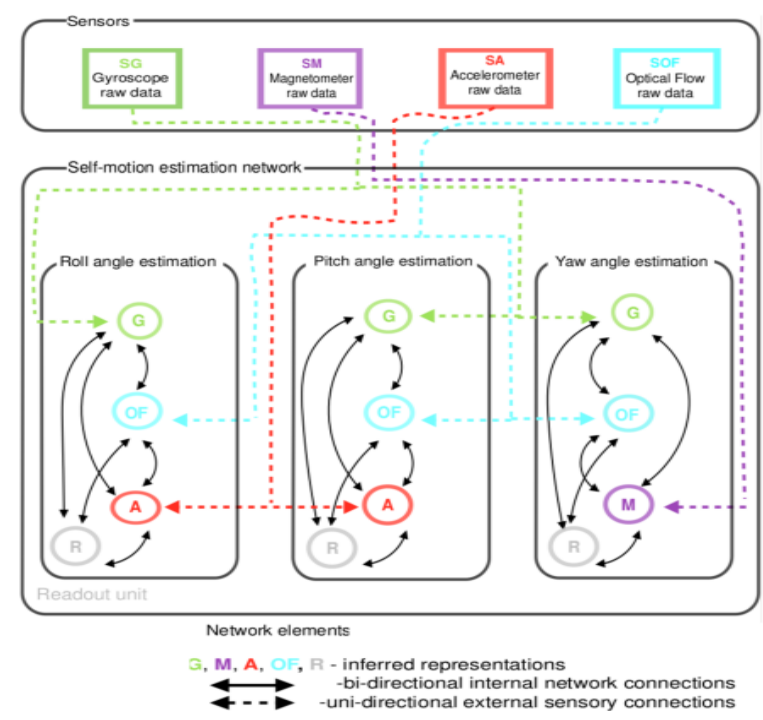

(a)
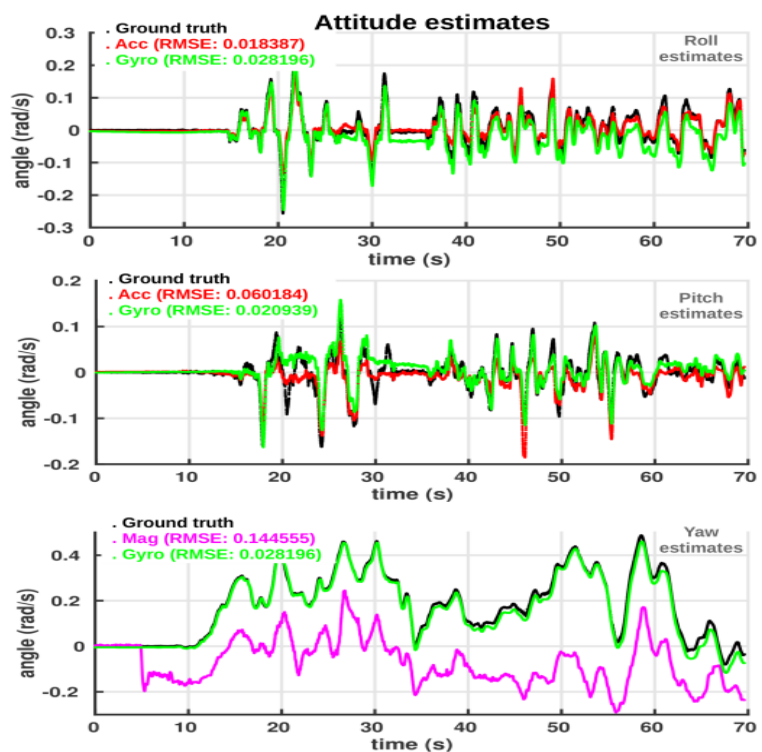

(b)

Figure 11. Network instantiation for 3D egomotion estimation: a decoupled view analysis. a) Learned relations; b) Estimation quality using learned relations.

As our results show, the model is able to extract the underlying data statistics without any prior information, such that the sensory data distribution is learned directly from the input data. Moreover, following the statistics of the data, the network allocates more neurons to represent areas in the sensory space with a higher density such that the cross-sensory relations are sharpened, visible in Figure 11(b). This is a very interesting feature that can be adopted for generic localization tasks. With this capability, manufacturing tools in a Cognitive Factory can react intelligently so that they can localize themselves in a factory working space. Regarding our method with state-of-the-art probabilistic sensory fusion mechanisms (i.e., Bayesian network), we address three key aspects: complexity, flexibility and robustness (see Table 1). 
Table 1. Comparison between state-of-the-art and our proposed model for sensor fusion

\begin{tabular}{|c|c|c|}
\hline Criteria & Bayesian Network & Proposed Model \\
\hline Complexity & $\begin{array}{l}\text { large number of probabilities to apply } \\
\text { probabilistic inference }\end{array}$ & compute multiple simple update rules \\
\hline Flexibility & $\begin{array}{l}\text { requires parameters adjustments for additional } \\
\text { sensory modalities; adding sensors improves } \\
\text { performance but increases complexity }\end{array}$ & $\begin{array}{l}\text { sensor addition (adding more update } \\
\text { rules/constraints) is straightforward and without } \\
\text { complexity increase }\end{array}$ \\
\hline Robustness & $\begin{array}{l}\text { dedicated means to detect failures, not generally } \\
\text { applicable; challenges in assigning probabilities in } \\
\text { an uncertain context }\end{array}$ & $\begin{array}{l}\text { abnormal sensor activity can be detected and } \\
\text { penalized by adapting } \eta \text { (e.g. the influence of that } \\
\text { sensor in the global estimate) }\end{array}$ \\
\hline
\end{tabular}
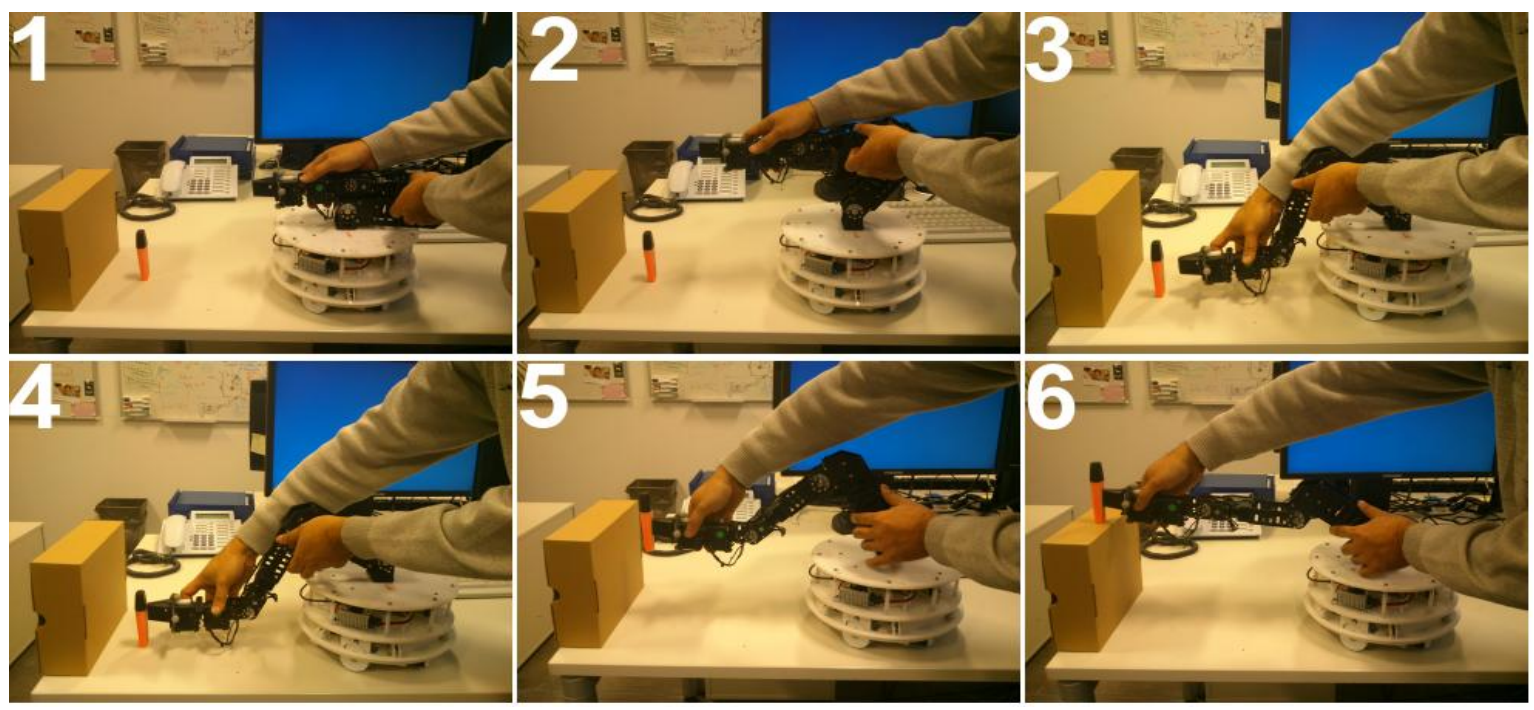

Figure 12. Guiding the robotic arm to follow a trajectory
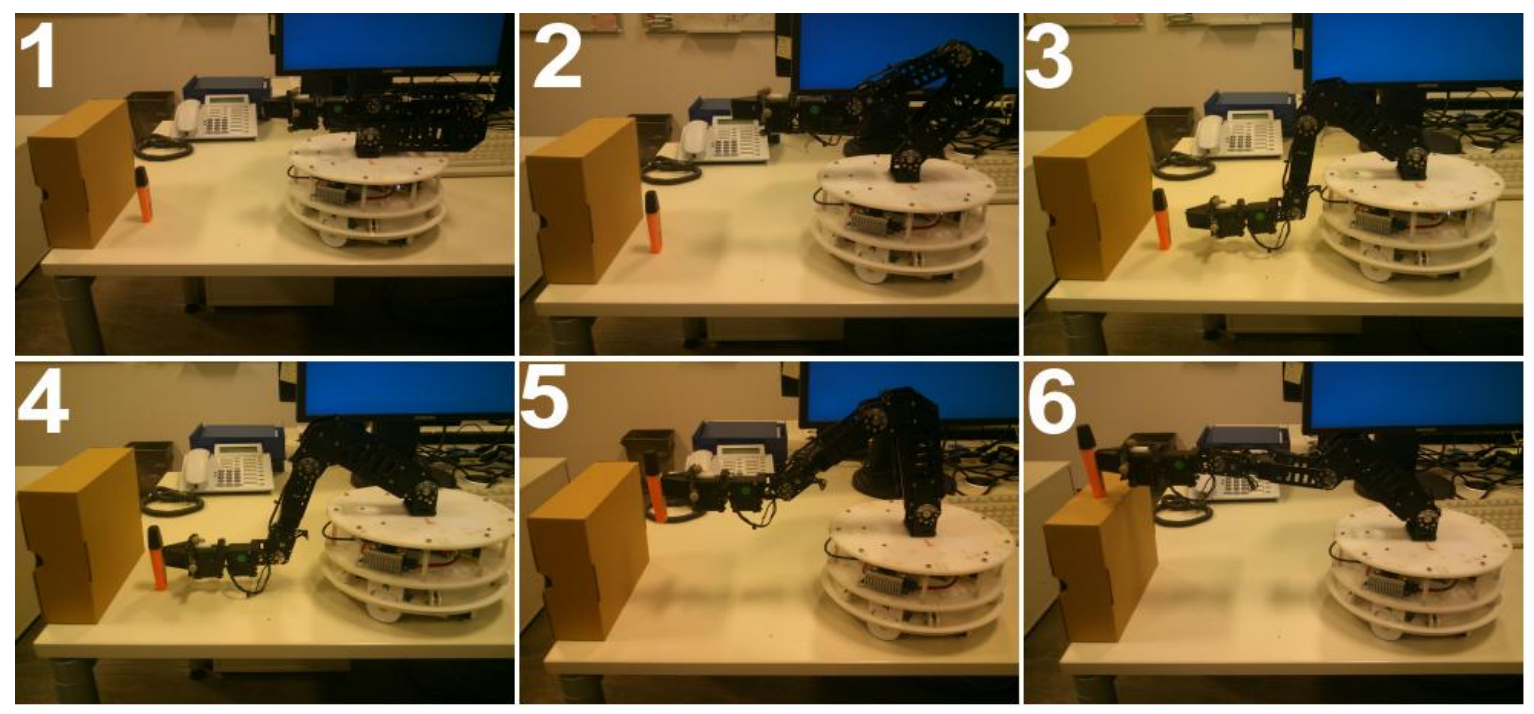

Figure 13. Robotic arm executes the trajectory it learned before

In section 3.2 we described one example of our proposed method to embody cognitive intelligence on a hybrid robotic platform. To use the $\mathrm{PbD}$ paradigm for generating the skill, we need to provide several demonstrations from which the trajectory of the movement can be learned. Each demonstration trajectory is fed into the regression network and the parameter of the network will be updated accordingly.
In general, any sequential actions can be performed and learned by using this $\mathrm{PbD}$ paradigm. As an example for a complex trajectory, we "guided" the robotic arm to pick up an object from one position and then place it on another position. This scenario is depicted in Figure 12. After several demonstrations, we perform the regression using the same mechanism shown in Figure 9. The estimated trajectory is then sent to the robot's kinematic controller. The snapshots of this run after learning the trajectory is depicted in Figure 13. 
From our experiment, the model-based learning applied to our hybrid robot was successfully implemented. From this result, we argue that we can extend our method to a more complex learning process in a dynamic environment of a Cognitive Factory. This is because once the robot learns the skill (i.e. the trajectory); it can use the standard scaling procedure to get different effects such as the different start and goal positions. At this point, we can see that our method has opened wider research challenges for further development of a Cognitive Factory. We regard these challenges as opportunity for our future work.

\section{Conclusion}

This paper presents a fundamental concept of delivering theories from neuroscience into engineering tasks that are suitable to build modern manufacturing systems. There are two important aspects that will play important roles in future manufacturing systems: changeability and human-machine collaboration. The first aspect, changeability, concerns with the ability of production tools to reconfigure themselves to the new manufacturing settings, possibly with unknown information a priori, while maintaining their reliability at lowest cost. The second aspect, human-machine collaboration, emphasizes on the ability of production tools to put themselves on the position as humans' co-workers. The interplay between these two aspects will not only determine the economical accomplishment of a manufacturing process, but it will also shape the future of the technology itself. To address this future challenge of manufacturing systems, we propose to embody cognitive intelligence on manufacturing components such that they become subjects of a Cognitive Factory. In this concept, machines and processes are equipped with cognitive capabilities in order to allow them to assess and increase their scope of operation autonomously, while maintaining their ability to work cooperatevely with humans. We introduced our method to achieve the goal of Cognitive Factory and gave two fundamental examples that exemplify the notion of brain-inspired information processing. Our method is inspired by the working mechanism of the human's brain; it works by harnessing the reasoning capabilities of cognitive architecture. By utilizing such an adaptive reasoning mechanism, we envision the future manufacturing systems with cognitive intelligence. By employing such a cognitive intelligence on manufacturing components, we will achieve the primary goals of Cognitive Factory: changeability and human-machine collaboration.

\section{Acknowledgements}

The authors would like to thank the German cluster of excellence CoTeSys and the Institute of Automatic Control Engineering at Technische Universität München, Germany, for the research opportunity and discussions with the experts' members (see also www.cotesys.org). The foundations of this work have been laid during workshops such as the Telluride Neuromorphic Cognition Engineering Workshop (see www.neuromorphs.net), the CapoCaccia Cognitive Neuromorphic Engineering Workshop (see capocaccia.iniforum.ch), and workshops sponsored by the
Munich Bernstein Center for Computational Neuroscience (see www.bccn-munich.de).

\section{References}

1. Tchoffa, D., Figay, N., Ghodous, P., Exposito, E., Kermad, L., Vosgien, T., and El Mhamedi, A., Digital Factory System for Dynamic Manufacturing Network Supporting Networked Collaborative Product Development, Data \& Knowledge Engineering, In Press (Available online 8 March 2016).

2. Essers, M.S. and Vaneker, T.H.J., Evaluating a Data Distribution Service System for Dynamic Manufacturing Environments: A Case Study, Procedia Technology, 15, 2014, pp. 622-631.

3. Babiceanu, R.F. and Seker, R., Big Data and Virtualization for Manufacturing Cyber-Physical Systems: A Survey of the Current Status and Future Outlook, Computers in Industry, 81, Sep 2016, pp. 128-137.

4. ElMaraghy, H.A., Flexible and Reconfigurable Manufacturing Systems Paradigms, International Journal of Flexible Manufacturing Systems, 17(4), Oct 2005, pp. 261-276.

5. Da Silva, R.M., Junqueira, F., Filho, D.J.S., and Miyagi, P.E., Control Architecture and Design Method of Reconfigurable Manufacturing Systems, Control Engineering Practice, 49, Apr 2016, pp. 87-100.

6. Vokurka, R.J. and O'Leary-Kelly, S.W., A Review of Empirical Research on Manufacturing Flexibility, Journal of Operations Management, 18(4), Jun 2000, pp. 485-501.

7. D’Souza, D.E. and Williams, F.P., Toward a Taxonomy of Manufacturing Flexibility Dimensions, Journal of Operations Management, 18(5), Aug 2000, pp. 577593.

8. Shi, D. and Daniels, R.L., A Survey of Manufacturing Flexibility: Implications for E-Business Flexibility, IBM Systems Journal, 42(3), 2003, pp. 414-427.

9. Wiendahl, H.P., ElMaraghy, H.A., Nyhuis, P., Zah, M.F., Wiendahl, H.H., Duffie, N., and Brieke, M., Changeable Manufacturing - Classification, Design and Operation, CIRP Annals - Manufacturing Technology, 56(2), 2007, pp. 783-809.

10. Beetz, M., Buss, M., and Wollherr, D., Cognitive Technical Systems - What Is the Role of Artificial Intelligence?, Proc. of $30^{\text {th }}$ Annual German Conference on Artificial Intelligence (KI 2007): Advances in Artificial Intelligence, LNCS: 4667, Osnabrück (Germany), Sep 2007, pp. 19-42.

11. Putzer, H., and Onken, R., COSA - A Generic Cognitive System Architecture Based on a Cognitive Model of Human Behavior, Cognition, Technology \& Work, 5(2), Jun 2003, pp. 140-151.

12. Shea, K., Engelhard, M., Ertelt, C., and Hoisl, F., A Framework for a Cognitive Design-To-Fabrication System, Proc. of $7^{\text {th }}$ International Symposium on Tools and Methods of Competitive Engineering (TMCE), Izmir (Turkey), Apr 2008, pp. 1185-1200.

13. Brachman, R.J., Systems That Know What They're Doing, IEEE Intelligent Systems, 17(6), Nov-Dec 2002, pp. 67-71. 
14. Hoc, J.M., Towards a Cognitive Approach to Human Machine Cooperation in Dynamic Situations, International Journal of Human-Computer Studies, 54(4), 2001, pp. 509-540.

15. Swindale, N.V., How Different Feature Spaces May Be Represented in Cortical Maps, Network, 15(4), Nov 2004, pp. 217-242.

16. Bressler, S.L., Understanding Cognition through LargeScale Cortical Networks, Current Directions in Psychological Science, 11(2), Apr 2002, pp. 58-61.

17. Bressler, S.L. and Tognoli, E., Operational Principles of Neurocognitive Networks, International Journal of Psychophysiology, 60(2), May 2006, pp. 139-148.

18. Pouget, A., Beck, J.M., Ma, W.J., and Latham, P.E., Probabilistic Brains: Knowns and Unknowns, Nature Neuroscience, 16(9), Aug 2013, pp. 1170-1178.

19. Michler, F., Eckhorn, R., and Wachtler. T., Using Spatiotemporal Correlations to Learn Topographic Maps for Invariant Object Recognition, Journal of Neurophysiology, 102(2), Aug 2009, pp. 953-964.

20. Liberman, N. and Trope, Y., The Psychology of Transcending the Here and Now, Science, 322(5905), Nov 2008, pp. 1201-1205.

21. Schaal, S. and Schweighofer, N., Computational Motor Control in Humans and Robots, Current Opinion in Neurobiology, 15(6), Dec 2005, pp. 675-682.

22. Schaal, S., Is Imitation Learning the Route to Humanoid Robots?, Trends in Cognitive Sciences, 3(6), Jun 1999, pp. 233-242.

23. Bullmore, E.T. and Bassett, D.S., Brain Graphs: Graphical Models of the Human Brain Connectome, Annual Review of Clinical Psychology, 7, 2011, pp. 113-140.

24. Toga, A.W., Clark, K.A., Thompson, P.M., Shattuck, D.W., and Van Horn, J.D., Mapping the Human Connectome, Neurosurgery, 71(1), Jul 2012, pp. 1-5.
25. Cao, M., Wang, J.H., Dai, Z.J., Cao, X.Y., Jiang, L.L., Fan, F.M., Song, X.W., Xia, M.R., Shu, N., Dong, Q., Milham, M.P., Castellanos, F.X., Zuo, X.N., and He, Y., Topological Organization of the Human Brain Functional Connectome Across the Lifespan, Developmental Cognitive Neuroscience, 7, Jan 2014, pp. 76-93.

26. Danks, D., Unifying the Mind: Cognitive Representations as Graphical Models, The MIT Press, 2014.

27. Axenie, C. and Conradt, J., Cortically Inspired Sensor Fusion Network for Mobile Robot Egomotion Estimation, Robotics and Autonomous Systems, 71, Sep 2015, pp. 69-82.

28. Arleo, A. and Rondi-Reig, L., Multimodal Sensory Integration and Concurrent Navigation Strategies for Spatial Cognition in Real and Artificial Organisms, Journal of Integrative Neuroscience, 6(3), Sep 2007, pp. 327-366.

29. Sheets-Johnstone, M., Movement: The Generative Source of Spatial Perception and Cognition, Spatial Cognition, Spatial Perception: Mapping the Self and Space (eds. Dolins, F.L. and Mitchell, R.W.), Cambridge University Press, 2010, pp. 323-340.

30. Glodek, M., Honold, F., Geier, T., Krell, G., Nothdurft, F., Reuter, S., Schussel, F., Hornle, T., Dietmayer, K., Minker, W., Biundo, S., Weber, M., Palm, G., and Schwenker, F., Fusion Paradigms in Cognitive Technical Systems for Human-Computer Interaction, Neurocomputing, 161, Aug 2015, pp. 17-37.

31. Ijspeert, A.J., Nakanishi, J., and Schaal, S., Movement Imitation with Nonlinear Dynamical Systems in Humanoid Robots, Proc. of IEEE International Conference on Robotics and Automation (ICRA), Vol. 2, Washington (USA), May 2002, pp. 1398-1403.

32. Axenie, C. and Conradt, J., Learning Sensory Correlations for 3D Egomotion Estimation, Proc. of $4^{\text {th }}$ International Conference on Biomimetic and Biohybrid Systems (Living Machines 2015), LNCS: 9222, Barcelona (Spain), Jul 2015, pp. 329-338. 